\title{
The correlation of heart rate between natural sleep and dexmedetomidine sedation
}

\author{
Donghee Kang, Changwoo Lim, Dong-jin Shim, Huiyoung Kim, Ji-wook Kim, \\ Hyung-joo Chung, Yusom Shin, Joo-Duck Kim, and Sie Jeong Ryu
}

Department of Anesthesiology and Pain Medicine, Kosin University College of Medicine, Busan, Korea

Background: Sedation by dexmedetomidine, like natural sleep, often causes bradycardia. We explored the nature of heart rate (HR) changes as they occur during natural sleep versus those occurring during dexmedetomidine sedation.

Methods: The present study included 30 patients who were scheduled to undergo elective surgery with spinal anesthesia. To assess HR and sedation, a pulse oximeter and bispectral index (BIS) monitor were attached to the patient in the ward and the operating room. After measuring HR and BIS at baseline, as the patients slept and once their BIS was below 70, HR and BIS were measured at 5-minute intervals during sleep. Baseline HR and BIS were also recorded before spinal anesthesia measured at 5-minute intervals after dexmedetomidine injection.

Results: During natural sleep, HR changes ranged from 2 to 19 beats $/ \mathrm{min}$ (13.4 \pm 4.4 beats $/ \mathrm{min})$, while in dexmedetomidine sedation, HR ranged from 9 to 40 beats $/ \mathrm{min}(25.4 \pm 8.5$ beats $/ \mathrm{min})$. Decrease in HR was significantly correlated between natural sleep and dexmedetomidine sedation $\left(\mathrm{R}^{2}=0.41, \mathrm{P}<0.001\right)$. The lowest HR was reached in 66 min during natural sleep ( 59 beats $/ \mathrm{min}$ ) and in $13 \mathrm{~min}$ with dexmedetomidine sedation ( 55 beats $/ \mathrm{min}$ ). The time to reach minimum HR was significantly different $(\mathrm{P}<0.001)$, but there was no difference in the lowest $\mathrm{HR}$ obtained $(\mathrm{P}=0.09)$.

Conclusions: There was a correlation between the change in HR during natural sleep and dexmedetomidine sedation. The bradycardia that occurs when using dexmedetomidine may be a normal physiologic change, that can be monitored rather than corrected.

Keywords: Dexmedetomidine; Heart rate; Sedatives; Sleep; Spinal anesthesia.

Corresponding author: Sie Jeong Ryu, M.D., Ph.D.

Department of Anesthesiology and Pain Medicine, Kosin University College of Medicine, 262 Gamcheon-ro, Seo-gu, Busan 49267, Korea Tel: 82-51-990-6474, Fax: 82-51-254-2504

Email: siejeong@ns.kosinmed.or.kr

ORCID: https://orcid.org/0000-0002-0677-4168

Received: July 18, 2018.

Revised: September 13, 2018 (1st); November 19, 2018 (2nd).

Accepted: November 19, 2018.

Korean J Anesthesiol 2019 April 72(2): 164-168

https://doi.org/10.4097/kja.d.18.00208

\section{Introduction}

Dexmedetomidine is a receptor agonist highly selective for the $\alpha_{2}$-adrenergic receptor, the affinity ratio for $\alpha_{1}: \alpha_{2}$ adrenergic receptors of $1: 1600$ [1]. This agonistic behavior upon the a2-adrenergic receptor leads to sedative, analgesic, and anxiolytic effects, making dexmedetomidine useful for sedation and analgesia in the field of anesthesia. Dexmedetomidine reduces nausea, vomiting, and anxiety, along with anti-shivering effects; it is anesthetic sparing without causing respiratory depression [2-4]. However, by acting on the $\alpha_{2}$-adrenergic receptor of the sympathetic ganglia, dexmedetomidine can cause severe bradycardia and hypotension [5].

Dexmedetomidine sedation mimics the natural sleep state, showing an electroencephalography (EEG) signal pattern sim-

(c) This is an open-access article distributed under the terms of the Creative Commons Attribution Non-Commercial License (http://creativecommons.org/ licenses/by-nc/4.0/), which permits unrestricted non-commercial use, distribution, and reproduction in any medium, provided the original work is properly cited. 
ilar to that of second stage non-rapid eye movement (NREM). The awakening pattern from sedation is comparable to that of awakening in natural sleep [6]. In addition, cardiovascular and respiratory patterns of dexmedetomidine sedation are similar to those of natural sleep [7]. During natural sleep, the vagus nerve activity increases, resulting in increased heart rate variability. During NREM sleep, the heart rate slows down, and blood pressure decreases. With dexmedetomidine sedation, mean arterial pressure increases by $7 \%$ and heart rate declines by $16 \%-18 \%$ during initial loading dose; then mean arterial pressure decreases and heart rate remains reduced during continuous infusion [8].

The purpose of this study was to evaluate the relationship of heart rate variability between natural sleep and dexmedetomidine sedation. Furthermore, we attempted to confirm that bradycardia occurring with dexmedetomidine use might be a normal physiological change.

\section{Materials and Methods}

A total of thirty patients participated in this study. The demographic data of these patients are presented in Table 1. The present study was approved by the clinical research ethics committee. Patients identified as American Society of Anesthesiologist physical status class I-II for elective surgery with spinal anesthesia were included. Patients with a history of heart disease, respiratory disease, obesity, or sleep apnea were excluded from the study.

On the day before surgery, a pulse oximeter (Suresigns VM6, Philips, USA) was attached to the patient to measure heart rate and oxygen saturation, while a BIS monitor (BIS VISTA, Aspect Medical Systems, USA) determined bispectral index (BIS) during natural sleep. Real-time data were automatically recorded for 90 min after BIS values reached 70 or less following the initiation of sleep. Baseline heart rate and BIS were defined 5 min before the initiation of sleep.

\begin{tabular}{lc} 
Table 1. Patient Characteristics \\
\hline Sex $(\mathrm{M} / \mathrm{F})$ & $17 / 13$ \\
Age $(\mathrm{yr})$ & $39.8 \pm 12.6$ \\
Height $(\mathrm{cm})$ & $166.8 \pm 8.7$ \\
Weight $(\mathrm{kg})$ & $64.6 \pm 9.0$ \\
Level of spinal anesthesia & \\
T7-8 & 10 \\
T9-10 & 15 \\
T11-12 & 5 \\
Dexmedetomidine total dose $(\mu \mathrm{g})^{*}$ & $81.4 \pm 11.1$ \\
Dexmedetomidine total infusion time $(\mathrm{min})$ & $99.0 \pm 23.3$
\end{tabular}

Values are presented as number or mean \pm SD. ${ }^{*}$ Total amount of dexmedetomidine during surgery.
The next day, the same two monitoring devices were used during operation. Pre-anesthetic medication was not administered. For spinal anesthesia, 10-12 mg of heavy bupivacaine was used, according to the patient's characteristics, aiming at T10 anesthesia. The level of the spinal anesthesia was checked using the pinprick test. After confirming the level of spinal anesthesia, loading dose of dexmedetomidine $(1 \mu \mathrm{g} / \mathrm{kg})$ was administered over $10 \mathrm{~min}$ for sedation. The maintenance dose of dexmedetomidine was $0.2 \mu \mathrm{g} / \mathrm{kg} / \mathrm{h}$ and the infusion rate was adjusted to maintain a BIS of 50-70. If the heart rate decreased to less than 40 beats/min, $0.5 \mathrm{mg}$ of atropine was administered intravenously. If the blood pressure decreased by more than $30 \%$ or the systolic blood pressure fell below $100 \mathrm{mmHg}, 5 \mathrm{mg}$ of ephedrine was administered.

The baseline value was measured in a stable state before spinal anesthesia administration. After spinal anesthesia, heart rate and BIS were measured every 5 min starting from the beginning of the dexmedetomidine loading dose. The time at which heart rate and BIS values reached their lowest values was recorded, along with the total amount of dexmedetomidine administered during surgery.

Statistical analysis was performed using the SPSS (version 24.0, IBM, USA) statistical program. For comparison of the lowest heart rate and BIS value, the corresponding paired T test was performed. Simple regression analysis was performed to determine the relationship of heart rate variability between the natural sleep and dexmedetomidine sedation. The results are expressed as mean $\pm \mathrm{SD}$, and statistical significance was defined as $\mathrm{P}<0.05$.

\section{Results}

A total of 30 patients participated in this study. The demographic data of these patients are presented in Table 1.

Table 2. Comparisons of Characteristics between Natural Sleep and Dexmedetomidine Sedation

\begin{tabular}{lllr}
\hline & Natural sleep & $\begin{array}{c}\text { Dexmede- } \\
\text { tomidine } \\
\text { sedation }\end{array}$ & P value \\
\hline Heart rate (beats/min) & & & \\
$\quad$ Baseline & $72.0 \pm 13.4$ & $80.0 \pm 15.1$ & $<0.001$ \\
$\quad$ Lowest point & $58.6 \pm 11.8$ & $54.6 \pm 11.3$ & 0.090 \\
$\quad$ Time (min) & $66.3 \pm 9.7$ & $12.5 \pm 6.8$ & $<0.001$ \\
Bispectral index & & & \\
$\quad$ Baseline & $93.7 \pm 2.8$ & $94.1 \pm 4.8$ & 0.682 \\
$\quad$ Lowest point & $45.0 \pm 10.9$ & $45.7 \pm 11.6$ & 0.786 \\
$\quad$ Time (min) & $34.0 \pm 13.7$ & $31.2 \pm 10.7$ & 0.374 \\
\hline
\end{tabular}

Values are presented as mean \pm SD. HR: heart rate. Time: Time to maximal decrease of heart rate and bispectral index. 


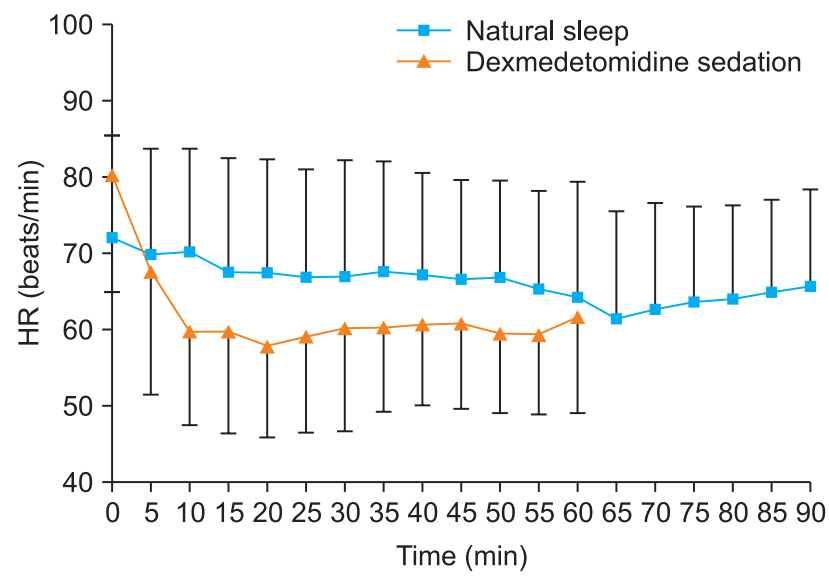

Fig. 1. Change of heart rate (HR) during natural sleep and dexmedetomidine sedation over time.

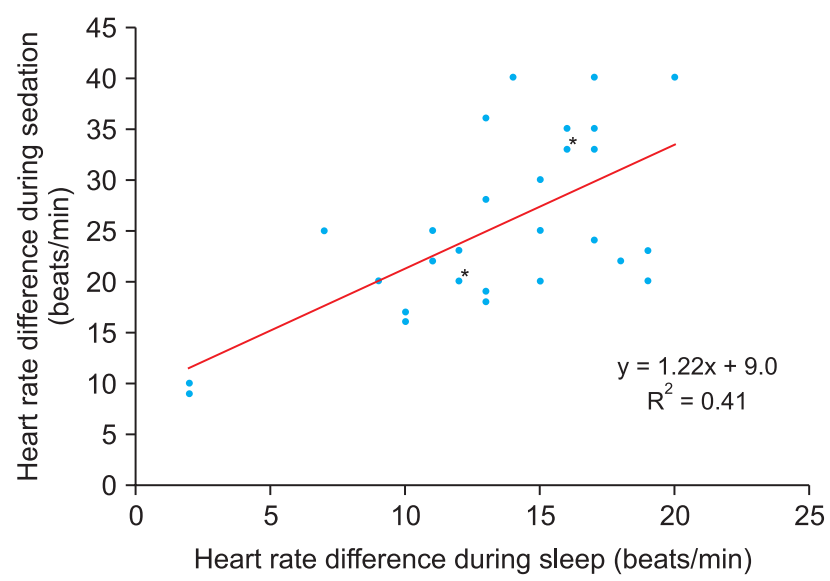

Fig. 2. Linear relationship between heart rate changes during natural sleep and during dexmedetomidine sedation. ${ }^{*}$ The data obtained for both conditions are superimposed.

The baseline heart rate was significantly different between natural sleep and dexmedetomidine sedation (72.0 \pm 13.4 beats/ min vs. $80.0 \pm 15.1$ beats $/ \mathrm{min}$, respectively) $(\mathrm{P}<0.001$, Table 2$)$. The lowest heart rate was $58.6 \pm 11.8$ beats $/ \mathrm{min}$ in natural sleep and $54.6 \pm 11.3$ beats/min in dexmedetomidine sedation which was not statistically significant $(P=0.09)$. The time to reach the lowest point was $66.3 \pm 9.7 \mathrm{~min}$ in natural sleep and $12.5 \pm$ $6.8 \mathrm{~min}$ in dexmedetomidine sedation which was significantly different $(\mathrm{P}<0.001$, Fig. 1). The difference in heart rate between baseline and the point of lowest heart rate was $13.4 \pm 4.4$ beats/ min in natural sleep and $25.4 \pm 8.5$ beats/min in dexmedetomidine sedation; this difference was thought to be due to the difference in base values. There was a significant correlation in reduction of heart rate between the natural sleep and dexmedetomidine sedation $\left(\mathrm{R}^{2}=0.41, \mathrm{P}<0.001\right.$, Fig. 2$)$.

BIS levels during natural sleep declined to less than 70 be-

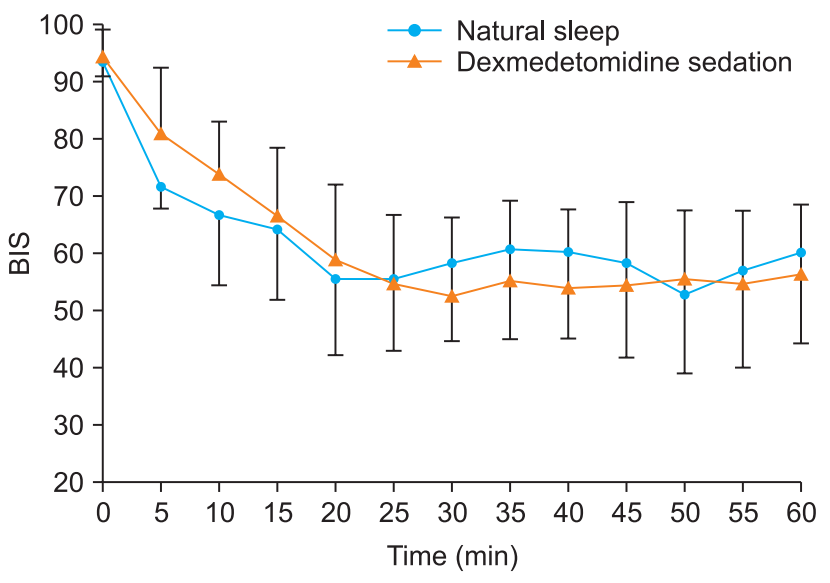

Fig. 3. Change of bispectral index (BIS) values during natural sleep and dexmedetomidine sedation over time.

tween 10 and 15 min. During sedation, BIS levels decreased to less than 70 between 5 and 10 min after the loading dose was injected. Subsequent BIS levels remained similar in both natural sleep and dexmedetomidine sedation (Fig. 3).

\section{Discussion}

Dexmedetomidine has a high selectivity for $\alpha_{2}$-adrenergic receptor, acting on the $\alpha_{2 A}, \alpha_{2 B}$-adrenergic receptors. It acts on the $\alpha_{2 B}$-adrenergic receptor to cause vasoconstriction and an increase in blood pressure in the early stage of injection [9]. In the present study, heart rate decreased when injecting dexmedetomidine, reaching a minimum heart rate similar to natural sleep, though in a significantly shorter period of time. The difference in the time point when the blood pressure reached the lowest level was due to the rapid increase in plasma concentration when the dexmedetomidine loading dose was injected. As a result, the blood pressure is temporarily increased, and the heart rate is likely decreased as a natural reflex. Continuous infusion of dexmedetomidine causes the blood pressure to decrease inversely, but the heart rate remains lower than the baseline [10]. In the present study, the heart rate decreased to the lowest value when the loading dose was injected, and then the heart rate remained constant when the maintenance dose was injected. However, the baseline heart rate before spinal anesthesia was higher than that of natural sleep which is thought to be the result of a patient's anxiety from being in the operating room without premedication such as midazolam.

In the present study, the heart rate reached its lowest point in $66.3 \pm 9.7 \mathrm{~min}$ during natural sleep, and the heart rate tended to rise around $80 \mathrm{~min}$. This change in heart rate during natural sleep may be a result of the change in the sympathetic-parasympathetic balance [11]. During natural sleep, the sympathetic 
nervous system does not show large fluctuations, but there are changes in the activity of the parasympathetic nerves. The balance between sympathetic nerves and parasympathetic nerves during awakening shifts toward parasympathetic as the level of sleep progresses, and heart rate gradually slows. However, at the REM sleep stage, the activity of the parasympathetic system decreases, and the heart rate rises to a level similar to the awakened state [12].

After a rapid heart rate decline during sedation with dexmedetomidine, the heart rate remained at a level similar to the lowest heart rate observed during natural sleep. The greater the decrease in heart rate during natural sleep, the greater the decrease in heart rate when sedated with dexmedetomidine, and this amount of reduction in heart rate was statistically related $\left(\mathrm{R}^{2}\right.$ $=0.41, \mathrm{P}<0.001)$. Studies have shown that EEG patterns during dexmedetomidine sedation are similar to those during natural sleep $[13,14]$. In the present study, similar patterns of BIS levels were observed during natural sleep and sedation with dexmedetomidine. These results show that dexmedetomidine not only produces sedation with characteristics similar to those of natural sleep, but also results in changes of heart rate comparable to those of natural sleep.

Bradycardia is a common complication of dexmedetomidine use [5]. Complications leading to death have been reported when dexmedetomidine was used in conjunction with other anesthetic agents [15]; to date, there have been no reports of death related to dexmedetomidine alone. While dexmedetomidine use frequently reduces heart rate, it does not cause major problems. Therefore, as confirmed in the present study, it may be sufficient to simply monitor dexmedetomidine-associated bradycardia without immediate correction with anticholinergic drugs such as atropine. However, attention should be paid to other factors that may affect heart rate. When dexmedetomidine is used in conjunction with other medications that can lower heart rate, such as propofol and fentanyl, the risk of severe bradycardia is greater [15]. If spinal anesthesia reaches above the above T4 level, the cardioaccelerator fibers may be blocked and cause bradycardia. If this happens in association with dexmedetomidine, serious bradycardia may occur. Therefore, when dexmedetomidine is used, careful attention should be paid to avoid inadvertent elevation of spinal anesthesia, or the use of dexmedetomidine should be reduced if the height of the anesthesia is unintentionally raised.

In the present study, the incidence of bradycardia, defined as a heart rate less than 40 beats/min, was relatively low at $5 \%$. Other studies have shown that the frequency of bradycardia varies from $10 \%$ to $30 \%$, depending on the amount of drug used [16-18] and their criteria for bradycardia. Additionally, because the dexmedetomidine infusion rate was controlled by maintaining the BIS value between 50 and 70, the probability of bradycar- dia was decreased. A better designed study should be conducted by adjusting the dosage of the drug in accordance with the BIS values as this may reduce bradycardia.

One limitation of this study was the possible effect of spinal anesthesia on heart rate, although the highest level of spinal anesthesia was T8. Second, the difference in baseline values between natural sleep and dexmedetomidine sedation makes interpretation of data more difficult. An anxiolytic agent such as midazolam was not administered before the operation, and it seems that as the patients came to the operating room with anxiety, this may have elevated their heart rate. Finally, in practice, it is difficult to measure heart rate during sleep. In our study, the patient's heart rate during sleep was measured using a portable patient monitor used for anesthesia, which is not widely available. Employing a simple, wearable smart band to evaluate sleep may compromise accuracy, but allow a larger set of meaningful measurements to be collected.

In conclusion, we found a correlation between the change in heart rate during natural sleep and dexmedetomidine sedation. The bradycardia that occurs when using dexmedetomidine may be a normal change. Therefore, the bradycardia that occurs when using dexmedetomidine should be observed rather than immediately treated so long as no other factors have caused it.

\section{Conflicts of Interest}

No potential conflict of interest relevant to this article was reported.

\section{Author Contributions}

Donghee Kang (Investigation; Project administration; Writing original draft)

Changwoo Lim (Data curation)

Dong-jin Shim (Investigation; Methodology)

Huiyoung Kim (Software)

Ji-wook Kim (Visualization)

Hyung-joo Chung (Formal analysis)

Yusom Shin (Writing - review \& editing)

Joo-Duck Kim (Writing - review \& editing)

Sie Jeong Ryu (Conceptualization; Project administration; Writing - review \& editing)

\section{ORCID}

Donghee Kang, https://orcid.org/0000-0001-6614-9244

Changwoo Lim, https://orcid.org/0000-0002-3070-1305

Dong-jin Shim, https://orcid.org/0000-0002-8704-529X

Huiyoung Kim, https://orcid.org/0000-0003-2330-1458

Ji-wook Kim, https://orcid.org/0000-0001-9944-2113 
Hyung-joo Chung, https://orcid.org/0000-0001-9545-3245

Yusom Shin, https://orcid.org/0000-0002-4829-0866
Joo-Duck Kim, https://orcid.org/0000-0002-9236-5183

Sie Jeong Ryu, https://orcid.org/0000-0002-0677-4168

\section{References}

1. Hayashi Y, Maze M. Alpha 2 adrenoceptor agonists and anaesthesia. Br J Anaesth 1993; 71: 108-18.

2. Venn RM, Hell J, Grounds RM. Respiratory effects of dexmedetomidine in the surgical patient requiring intensive care. Crit Care 2000; 4: 302-8.

3. Aantaa R, Kanto J, Scheinin M, Kallio A, Scheinin H. Dexmedetomidine, an alpha 2-adrenoceptor agonist, reduces anesthetic requirements for patients undergoing minor gynecologic surgery. Anesthesiology 1990; 73: 230-5.

4. Choi EK, Seo Y, Lim DG, Park S. Postoperative nausea and vomiting after thyroidectomy: a comparison between dexmedetomidine and remifentanil as part of balanced anesthesia. Korean J Anesthesiol 2017; 70: 299-304.

5. Ebert TJ, Hall JE, Barney JA, Uhrich TD, Colinco MD. The effects of increasing plasma concentrations of dexmedetomidine in humans. Anesthesiology 2000; 93: 382-94.

6. Maksimow A, Snapir A, Särkelä M, Kentala E, Koskenvuo J, Posti J, et al. Assessing the depth of dexmedetomidine-induced sedation with electroencephalogram (EEG)-based spectral entropy. Acta Anaesthesiol Scand 2007; 51: 22-30.

7. Hsu YW, Cortinez LI, Robertson KM, Keifer JC, Sum-Ping ST, Moretti EW, et al. Dexmedetomidine pharmacodynamics: part I: crossover comparison of the respiratory effects of dexmedetomidine and remifentanil in healthy volunteers. Anesthesiology 2004; 101: 1066-76.

8. Hall JE, Uhrich TD, Barney JA, Arain SR, Ebert TJ. Sedative, amnestic, and analgesic properties of small-dose dexmedetomidine infusions. Anesth Analg 2000; 90: 699-705.

9. Philipp M, Brede M, Hein L. Physiological significance of alpha(2)-adrenergic receptor subtype diversity: one receptor is not enough. Am J Physiol Regul Integr Comp Physiol 2002; 283: R287-95.

10. Dyck JB, Maze M, Haack C, Vuorilehto L, Shafer SL. The pharmacokinetics and hemodynamic effects of intravenous and intramuscular dexmedetomidine hydrochloride in adult human volunteers. Anesthesiology 1993; 78: 813-20.

11. Zemaityte D, Varoneckas G, Sokolov E. Heart rhythm control during sleep. Psychophysiology 1984; 21: 279-89.

12. Trinder J, Kleiman J, Carrington M, Smith S, Breen S, Tan N, et al. Autonomic activity during human sleep as a function of time and sleep stage. J Sleep Res 2001; 10: 253-64.

13. Huupponen E, Maksimow A, Lapinlampi P, Särkelä M, Saastamoinen A, Snapir A, et al. Electroencephalogram spindle activity during dexmedetomidine sedation and physiological sleep. Acta Anaesthesiol Scand 2008; 52: 289-94.

14. Su X, Meng ZT, Wu XH, Cui F, Li HL, Wang DX, et al. Dexmedetomidine for prevention of delirium in elderly patients after non-cardiac surgery: a randomised, double-blind, placebo-controlled trial. Lancet 2016; 388: 1893-902.

15. Bharati S, Pal A, Biswas C, Biswas R. Incidence of cardiac arrest increases with the indiscriminate use of dexmedetomidine: a case series and review of published case reports. Acta Anaesthesiol Taiwan 2011; 49: 165-7.

16. Bloor BC, Ward DS, Belleville JP, Maze M. Effects of intravenous dexmedetomidine in humans. II. Hemodynamic changes. Anesthesiology 1992; 77: 1134-42.

17. Elcicek K, Tekin M, Kati I. The effects of intravenous dexmedetomidine on spinal hyperbaric ropivacaine anesthesia. J Anesth 2010; 24: 5448.

18. Ahn EJ, Park JH, Kim HJ, Kim KW, Choi HR, Bang SR. Anticholinergic premedication to prevent bradycardia in combined spinal anesthesia and dexmedetomidine sedation: a randomized, double-blind, placebo-controlled study. J Clin Anesth 2016; 35: 13-9. 